\title{
Nonradioactive Analysis of Dynamic Protein Palmitoylation
}

\author{
Brent R. Martin ${ }^{1}$ \\ ${ }^{1}$ Department of Chemistry, University of Michigan, Ann Arbor, Michigan
}

\begin{abstract}
Methods to study protein $S$-palmitoylation dynamics have previously relied on metabolic labeling with $\left[{ }^{14} \mathrm{C}\right]$ palmitate, which requires additional safety precautions and long exposures. Nonradioactive alkynyl palmitate analogs have been developed for in-gel fluorescence detection and affinity purification. Cells metabolically labeled with the commercially available analog 17-octadynoic acid are lysed and then combined with azide-linked reporter tags for efficient conjugation by copper-catalyzed click chemistry in phosphate buffer. This approach has been demonstrated to label hundreds of endogenous palmitoylated proteins and is compatible with traditional pulse-chase methods. This protocol describes the reagents and procedures for labeling and detection of dynamic palmitoylation in mammalian cells. Curr. Protoc. Protein Sci. 73:14.15.1-14.15.9. (C) 2013 by John Wiley \& Sons, Inc.
\end{abstract}

Keywords: palmitoylation $\bullet$ click chemistry $\bullet$ post-translational modifications

Protein $S$-palmitoylation describes the thioester linkage of long-chain fatty acids to cysteine residues in proteins. Traditional methods for analyzing $S$-palmitoylation require metabolic labeling with $\left[{ }^{3} \mathrm{H}\right]$ palmitate, immunoprecipitation, and SDS-PAGE separation followed by long film exposures for days or weeks. New methods have been developed based on metabolic incorporation of alkynyl fatty acid analogs. The commercially available analog 17-octadecynoic acid (17-ODYA) is readily accepted by the endogenous cellular palmitoylation machinery and transferred to endogenous sites of modification. After metabolic labeling, the extent of protein palmitoylation can be monitored by simple copper-catalyzed click chemistry to azide-linked reporter tags for in-gel fluorescence analysis or affinity enrichment. This approach is especially useful for monitoring the dynamics of palmitoylation using classic pulse-chase approaches. This protocol describes the metabolic labeling and in-gel fluorescence analysis of dynamic palmitoylation in mammalian cells (Fig. 14.15.1).

\section{Materials}

Cultured mammalian cells

Culture medium (e.g., DMEM, Invitrogen, cat. no. 10564-011), $37^{\circ} \mathrm{C}$

DPBS, calcium and magnesium free (Invitrogen, cat. no. 14190-136), $37^{\circ}$ and $4^{\circ} \mathrm{C}$ (do not use EDTA)

17-ODYA labeling medium (see recipe), $37^{\circ} \mathrm{C}$

Palmitic acid chase medium (see recipe), $37^{\circ} \mathrm{C}$

Protein thioesterase inhibitor (select one):

Phenylmethylsulfonylfluoride (PMSF, Sigma, cat. no. P7626)

Hexadecylsulfonyl fluoride (HDSF, Calbiochem, cat. no. 373250)

Hexadecylfluorophosphonate (HDFP, available upon request, synthetic methods described in Martin et al., 2012)

$50 \mathrm{mM}$ copper(II) sulfate $\left(\mathrm{CuSO}_{4}\right.$, Sigma-Aldrich, cat. no. 451657$)$ in water

Current Protocols in Protein Science 14.15.1-14.15.9, August 2013

Published online September 2013 in Wiley Online Library (wileyonlinelibrary.com).

Post-

Translational Modification:

Specialized

Applications

14.15.1

Supplement 73 
$50 \mathrm{mM}$ tris 2-carboxyethyl phosphine (TCEP, Sigma-Aldrich, cat. no. 93284), freshly prepared in DPBS

$1 \times$ TBTA ligand solution (see recipe)

$1 \mathrm{mM}$ rhodamine-azide: tetramethylrhodamine (TAMRA) azide

[tetramethylrhodamine 5-carboxamido-(6-azidohexanyl), 5-isomer, Invitrogen, cat. no. T10182] in dimethyl sulfoxide (DMSO)

$50 \%$ (v/v) hydroxylamine (Alfa Aesar, cat no. B22202 or L16990), adjusted to $\mathrm{pH} 7$ in PBS

\section{Cell scraper}

Branson S-250A sonifier cell disruptor with 1/8-in. tapered microtip

Beckman Optima Max ultracentrifuge (or equivalent) with TLA-100.3 rotor

1.5-ml thick-walled ultracentrifuge tubes (Beckman, cat. no. 357448)

1.5-ml microcentrifuge tubes

Flatbed fluorescence gel scanner, Hitachi FMBIO II or equivalent

Additional reagents and equipment for protein quantification (UNIT 3.4), immunoprecipitation (UNIT 9.8, optional), SDS-PAGE (UNIT 10.1), scanning gels (UNIT 10.12), electroblotting (UNIT 10.7, optional), and detection of proteins on blots (UNIT 10.10, optional)

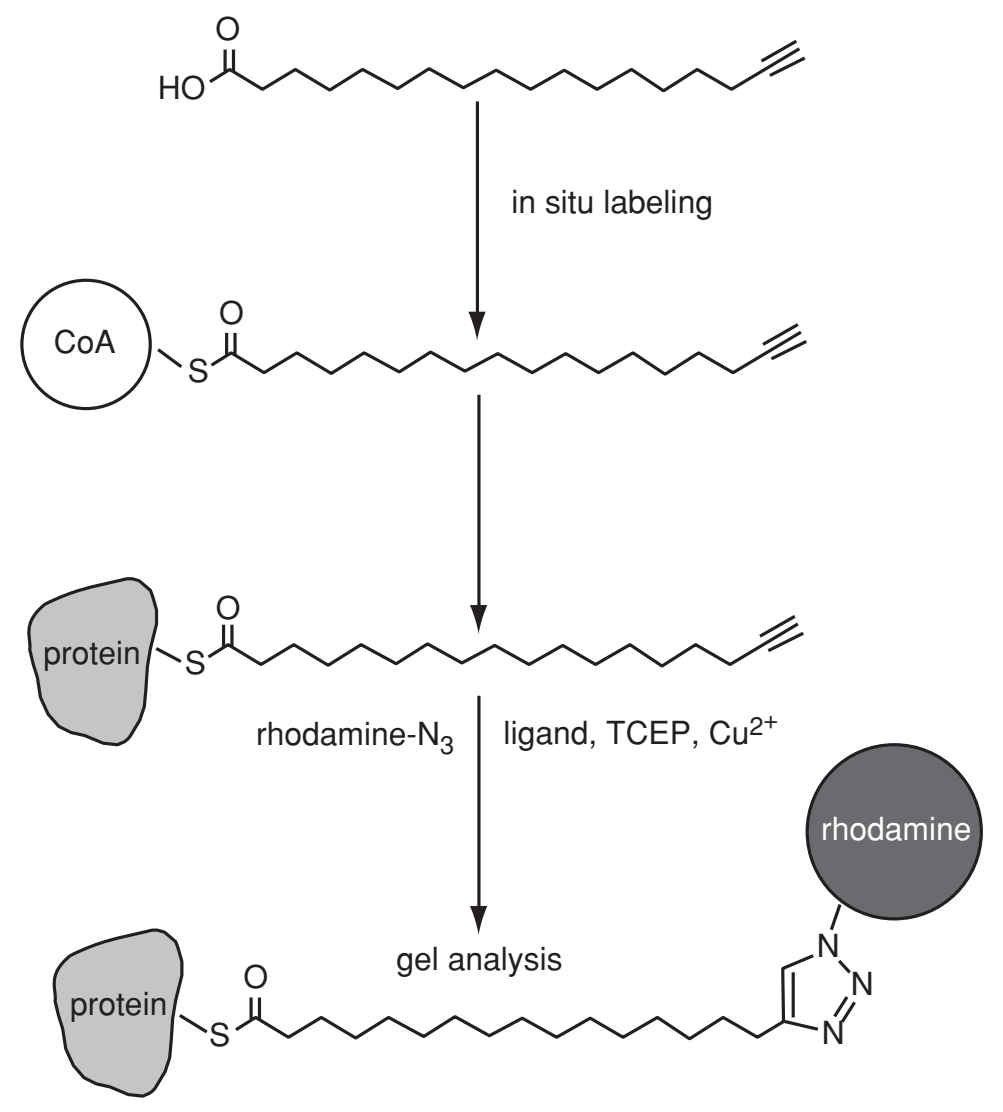

Figure 14.15.1 17-ODYA metabolic labeling and detection of palmitoylated proteins. The commercially available alkynyl fatty acid 17-ODYA is added to cells in culture. Over the course of several hours, the probe is conjugated to acetyl coenzyme $A(C o A)$ and can serve as a substrate for protein palmitoyl transferases. The cells are then lysed and mixed with click chemistry reagents. TCEP reduces $\mathrm{Cu}(\mathrm{II})$ to $\mathrm{Cu}(\mathrm{I})$, which is bound to the TBTA ligand. Addition of an azide-linked re-

Nonradioactive Analysis of Dynamic Protein Palmitoylation porter (e.g., rhodamine-azide or biotin-azide) facilitates click chemistry conjugation and triazole formation, covalently linking the reporter to 17-ODYA thioesters on proteins. The labeled palmitoylated proteins are separated by SDS-PAGE or affinity purified for mass spectrometry-based proteomics.

\subsection{2}


1. Grow mammalian cells to desired density in standard culture medium.

Membrane protein yields vary between cell lines, and the amount of protein needed depends on the application. For gel-based analysis, plan to harvest enough cells for $\sim 1 \mathrm{mg}$ proteome, which corresponds to $\sim 2 \times 10^{7}$ Jurkat cells in $20 \mathrm{ml}$ medium, or $\sim 4 \times 10^{6} 293$ T cells on a 6 -cm culture dish in $4 \mathrm{ml}$ medium.

2. Wash cells with warm DPBS $\left(37^{\circ} \mathrm{C}\right)$ to remove residual growth medium.

For suspension cells: Collect cells and centrifuge 5 min at $500 \times g$, room temperature. Decant supernatant and gently resuspend cell pellet in $10 \mathrm{ml}$ DPBS. Repeat once or twice.

For adherent cells: Gently aspirate medium and add a sufficient volume of DPBS to cover cells. Leave for $1 \mathrm{~min}$, then aspirate DPBS. Repeat twice. If cells detach, collect them and centrifuge as in step 2a, then add back to dish.

3. Add warm 17-ODYA labeling medium $\left(37^{\circ} \mathrm{C}\right)$ to the cells and incubate for the desired labeling time.

For suspension cells: Add enough medium to support log phase growth. For Jurkat cells, dilute to $1 \times 10^{6}$ cells $/ \mathrm{ml}$.

For adherent cells: Add labeling medium equal to the original volume.

It may be necessary to perform a time course to determine the optimal labeling time, but $2 \mathrm{hr}$ is typically sufficient for pulse-chase methods.

4. Optional: Add warm palmitic acid chase medium $\left(37^{\circ} \mathrm{C}\right)$ for predetermined lengths of time (e.g., 2, 4, and $6 \mathrm{hr}$ ).

5. Wash cells three times with cold DPBS $\left(4^{\circ} \mathrm{C}\right)$.

6. Harvest adherent cells by scraping. Pellet cells $2 \mathrm{~min}$ at $500 \mathrm{~g}, 4^{\circ} \mathrm{C}$, and place on ice.

Do not use trypsin to harvest adherent cells.

If desired, the cell pellet can be frozen at $-80^{\circ} \mathrm{C}$ and stored up to several months.

\section{Prepare membrane lysates}

7. Add an appropriate volume (typically, 0.5 to $1 \mathrm{ml}$ per $15-\mathrm{cm}$ plate of cells) of DPBS supplemented with serine protease inhibitor (1 mM PMSF, $0.2 \mathrm{mM} \mathrm{HDSF}$, or $0.02 \mathrm{mM}$ HDFP) and sonicate on ice to lyse cells.

These inhibitors inactivate potential protein thioesterase activities, although the lysosomal thioesterase PPT1 is resistant to PMSF. Avoid the use of Tris buffers or additives with free amines, which can interfere with click chemistry. Do not use EDTA.

8. In thick-walled ultracentrifuge tubes, separate soluble and insoluble fractions by ultracentrifuging $45 \mathrm{~min}$ at $100,000 \times \mathrm{g}, 4^{\circ} \mathrm{C}$.

9. Place pellet on ice and add fresh cold DPBS at an appropriate volume to yield $\sim 1-2 \mathrm{mg} / \mathrm{ml}$ proteome $(\sim 0.5 \mathrm{ml}$ for a standard $10-\mathrm{cm}$ plate of cells).

The supernatant can be discarded or transferred to a fresh 1.5-ml tube and set aside on ice for other applications.

10. Sonicate $10 \mathrm{sec}$ on ice for $5 \mathrm{sec}$ or until the pellet is dispersed.

11. Measure protein concentration by Bradford or equivalent assay (UNIT 3.4). Normalize concentration to $1.1 \mathrm{mg} / \mathrm{ml}$ with DPBS.

If desired, a specific target can be immunoprecpitated at this point. Use a standard immunoprecipitation protocol (e.g., UNIT 9.8) in phosphate buffer without EDTA.

Post-

Translational Modification:

Specialized

Applications

14.15.3 


\section{Perform click chemistry}

12. Transfer $50 \mu \mathrm{g}$ of the $1.1 \mathrm{mg} / \mathrm{ml}$ membrane lysate to a $1.5-\mathrm{ml}$ microcentrifuge tube and bring to $44 \mu \mathrm{l}$ total volume with PBS.

13. Add the following:

$1 \mu \mathrm{l} 50 \mathrm{mM} \mathrm{CuSO}_{4}$

$1 \mu 150 \mathrm{mM}$ TCEP

$3 \mu \mathrm{l} 1 \times$ TBTA ligand solution

$1 \mu 11 \mathrm{mM}$ rhodamine-azide.

Always use freshly prepared TCEP and do not reuse.

14. Vortex and let sit at room temperature for $30 \mathrm{~min}$, then vortex and let sit another $30 \mathrm{~min}$.

The copper precipitates proteins during this reaction in the absence of detergents. This reaction also works well with immunoprecipitated proteins on beads with continuous mixing.

15. Add $4 \times$ SDS gel loading buffer to $1 \times$ final.

Adding $\beta$-mercaptoethanol $(150 \mathrm{mM})$ to the loading buffer provides better resolution, but do not use dithiothreitol (DTT) and do not boil.

16. Remove half of the sample and place in a separate tube. To check for thioesterdependent labeling, add $1.25 \mu \mathrm{l}$ of $50 \%$ hydroxylamine, $\mathrm{pH} 7$, and incubate at room temperature for $1 \mathrm{hr}$ or boil $5 \mathrm{~min}$.

17. Separate all samples by standard SDS-PAGE (UNIT 10.1) on a Tris-glycine SDSpolyacrylamide gel and scan the gel fluorescence (UNIT 10.12).

Alternatively, if the lysate was reacted with biotin-azide, transfer the gel to nitrocellulose (UNIT 10.7), block with BSA (nonfat dry milk contains endogenous biotin), and probe with streptavidin-HRP for chemiluminescence detection (UNIT 10.10).

\section{REAGENTS AND SOLUTIONS}

Use deionized, distilled water in all recipes and protocol steps. For common stock solutions, see APPENDIX 2A; for suppliers, see SUPPLIERS APPENDIX.

\section{7-ODYA labeling medium}

Cell culture medium (e.g., DMEM, RPMI)

1\% penicillin-streptomycin-glutamine (Invitrogen, cat. no. 10378-016)

10\% dialyzed fetal bovine serum (e.g., Gemini Bio-Products, cat. no. 100-108)

$25 \mu \mathrm{M}$ 17-octadecynoic acid (17-ODYA, Cayman Chemical, cat. no. 90270) from $25 \mathrm{mM}$ stock in dimethyl sulfoxide (DMSO)

Sonicate or vortex to mix

Prepare fresh just before use and warm to $37^{\circ} \mathrm{C}$

\section{Palmitic acid chase medium}

Cell culture medium (e.g., DMEM, RPMI)

$1 \%$ penicillin-streptomycin-glutamine (Invitrogen, cat. no. 10378-016)

$10 \%$ dialyzed fetal bovine serum (e.g., Gemini Bio-Products, cat. no. 100-108)

$250 \mu \mathrm{M}$ palmitic acid from $500 \mathrm{mM}$ stock in DMSO

Nonradioactive Analysis of Dynamic Protein Palmitoylation

Sonicate thoroughly to dissolve palmitate acid to fine particulates

Prepare fresh just before use and warm to $37^{\circ} \mathrm{C}$

14.15.4 
$50 \times$ stock solution:

$8.85 \mathrm{mg}$ tris[(1-benzyl-1H-1,2,3-triazol-4-yl)methyl]amine (TBTA, SigmaAldrich, cat. no. 678937)

$200 \mu \mathrm{DMSO}$

Store up to 6 months at room temperature

$1 \times$ working solution:

$20 \mu \mathrm{l} 50 \times$ stock solution

$180 \mu \mathrm{DMSO}$

$800 \mu \mathrm{l} t$-butanol (Sigma-Aldrich, cat. no. 360538)

Store up to 6 months at room temperature

\section{COMMENTARY}

\section{Background Information}

The analysis of protein $S$-palmitoylation has traditionally utilized radioisotope labeling with $\left[{ }^{3} \mathrm{H}\right]$ palmitate (Schmidt et al., 1979; Schlesinger et al., 1980), which necessitates additional safety precautions for handling radioactivity and demands film exposures of days to weeks. Acyl biotin exchange (ABE) is a popular method to enrich and analyze palmitoylated proteins (Drisdel and Green, 2004; Roth et al., 2006; Kang et al., 2008) and involves thioester hydrolysis with hydroxylamine, followed by free thiol capture with disulfide-biotin derivatives or thiol resin (Forrester et al., 2011). This method is technically challenging. It requires complete reduction and alkylation of all thiols before hydroxylamine addition, which is accomplished by multiple washes and precipitation steps that alone can take several hours. Furthermore, this approach has no specificity for palmitoyl thioesters and leads to hydrolysis of all thioesters in a proteome sample. This leads to the nonspecific enrichment of other protein thioesters, including enzymes with lipoic acid cofactors and enzymes with stable acyl intermediates, such as ubiquitin ligases (Wan et al., 2007; Kang et al., 2008). Furthermore, ABE is not compatible with pulse-chase approaches to analyze palmitoylation stability and dynamics.

To circumvent these issues, bioorthogonal metabolically incorporated fatty acids have been introduced for rapid analysis of protein palmitoylation (Hang et al., 2007; Kostiuk et al., 2008; Martin and Cravatt, 2009). Since palmitoylation is a labile post-translational modification, there is a continual cycle of enzymatic and non-enzymatic hydrolysis followed by re-acylation. This provides a unique opportunity to metabolically incorporate modified acyl chains into existing proteins. The commercially available alkynyl fatty acid ana$\log 17-$ ODYA is incorporated by the endoge- nous cellular machinery to native palmitoylation sites. This nonradioactive analog enables pulse-chase labeling, which has led to identification of dynamic palmitoylation sites with rapid turnover kinetics (Zhang et al., 2010; Martin et al., 2012). These methods have been used to reveal that the majority of palmitoylation sites are relatively stable, while some palmitoylated proteins are enzymatically depalmitoylated and highly dynamic (Martin et al., 2012).

This unit provides a straightforward protocol for metabolic labeling and analysis of protein palmitoylation using click chemistry (Fig. 14.15.1). The choice of the azidemodified reporter depends on the downstream application (Charron et al., 2009; Martin and Cravatt, 2009). In-gel fluorescence is routinely used for simple analysis of global palmitoylation profiles. The choice of fluorophore depends on the availability of excitation wavelengths for the flatbed fluorescence gel scanner. Biotin-azide is also useful for streptavidin enrichment or blots. In comparison with ingel fluorescence approaches, streptavidin blots require additional steps for transfer and detection, and also highlight endogenous biotinylated proteins, such as carboxylase enzymes. These background biotinylated proteins can saturate the signal and prevent detection of signals that require high sensitivity. This issue can be resolved by pre-clearing lysates with avidin beads, but this adds additional purification steps. Biotin-azide is especially useful for enrichment of palmitoylated proteins for downstream proteomics applications for analysis of tryptic peptides (Martin and Cravatt, 2009; Yount et al., 2010).

\section{Critical Parameters and \\ Troubleshooting}

Several factors will determine the quality of 17-ODYA labeling and detection. Before
Post-

Translational Modification: Specialized Applications

14.15.5 


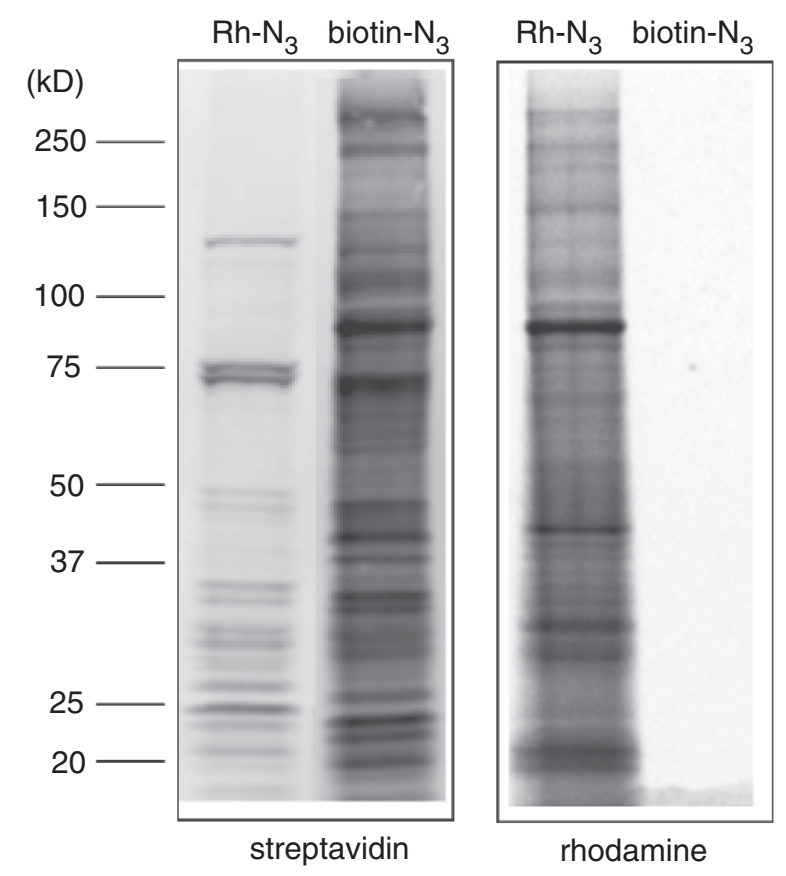

Figure 14.15.2 Comparison of rhodamine-azide $\left(\mathrm{Rh}-\mathrm{N}_{3}\right)$ and biotin-azide detection of 17-ODYA labeling. BW5147 cells were metabolically labeled with 17-ODYA for $4 \mathrm{hr}$, then lysed and fractionated. The insoluble fraction was reacted with $20 \mu \mathrm{M}$ rhodamine-azide or $400 \mu \mathrm{M}$ biotin-azide and separated on a $4 \%$ to $20 \%$ precast Tris-glycine SDS mini-gel. The gel was scanned on a Hitachi FMBioll flatbed fluorescence scanner to detect rhodamine fluorescence (right). It was then transferred to nitrocellulose and the blot was blocked with 5\% BSA, probed with Streptavidin 800 (LiCOR), and scanned using a LiCOR fluorescence scanner (left). This demonstrates the relative sensitivity of both detection approaches.

performing more challenging pulse-chase experiments, the concentration and time of 17-ODYA exposure required to achieve maximal metabolic incorporation should be optimized. Different cell lines have different metabolic rates, which can directly affect the efficiency of incorporation. After careful optimization of the labeling conditions, the chase conditions should also be carefully tested. The time and concentration of the pulse labeling is essential for achieving detectible palmitoylation turnover. If the labeling period is too long, 17-ODYA is metabolized into cellular lipid pools, and is especially difficult to wash out. There is a balance between longer labeling times required for robust detection and sufficient chase times to detect dynamic palmitoylation sites. Currently reported methods generally use 1 or $2 \mathrm{hr}$ of pulse labeling (Zhang et al., 2010; Martin et al., 2012). When using $\left[{ }^{3} \mathrm{H}\right]$ palmitate, much shorter pulse labeling protocols are especially useful for detecting rapid turnover (Ahearn et al., 2011). This sug- gests that similar rapid methods could be coupled with immunoaffinity enrichment of select targets for nonradioactive detection using 17ODYA.

During lysis, it is useful to include nonselective serine hydrolase inhibitors to block potential thioesterase activities. Reports have demonstrated that the lysosomal palmitoylprotein thioesterase 1 (PPT1) is resistant to PMSF inhibition, yet sensitive to HDSF inhibition (Das et al., 2000). This inhibitor is commercially available and potentially useful in samples with high PPT1 activities. Palmostatin B has also been shown to inhibit acylprotein thioesterases 1 and 2 (APT1, 2), and PPT1, which covers all three known candidate protein palmitoyl thioesterases (Dekker et al., 2010; Rusch et al., 2011). The nonselective lipase inhibitor HDFP has been demonstrated to sufficiently inhibit protein palmitoyl thioesterase activity in lysates by blocking more than 20 serine hydrolases, including PPT1 (Martin et al., 2012). It is important to 


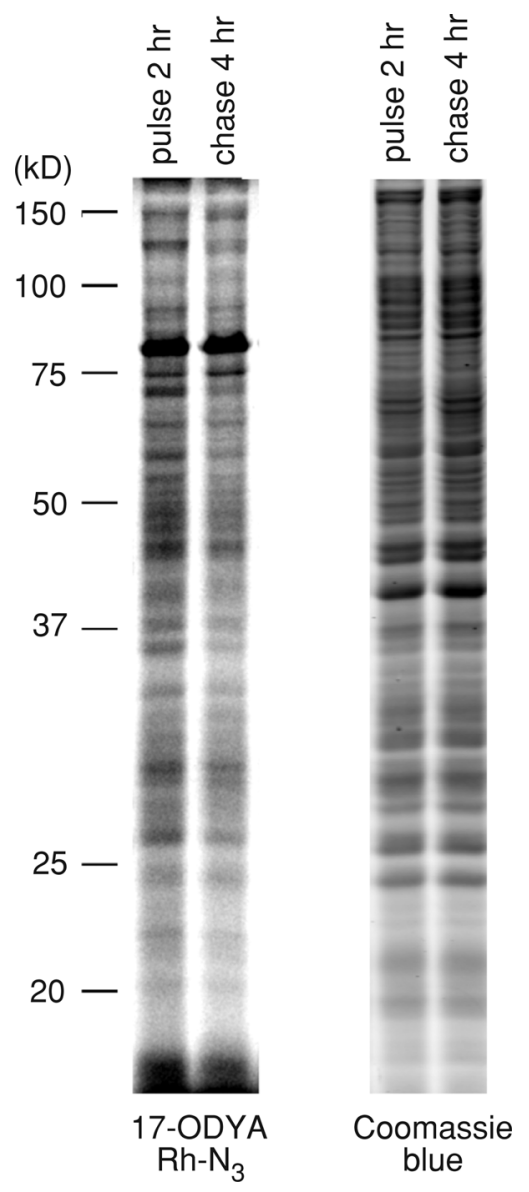

Figure 14.15.3 Pulse-chase analysis of dynamic protein palmitoylation. BW5147-derived T cell hybridoma cells were labeled with $20 \mu \mathrm{M}$ 17-ODYA for $2 \mathrm{hr}$ and an aliquot was washed and frozen. The remaining cells were washed and placed in medium containing $250 \mu \mathrm{M}$ palmitic acid for $4 \mathrm{hr}$, then washed and frozen. Cells pellets were lysed by sonication in the presence of $20 \mu \mathrm{M}$ HDFP, then separated into soluble and insoluble fractions by ultracentrifugation at $100,000 \times g$ for $45 \mathrm{~min}$. The insoluble fraction was diluted to $1 \mathrm{mg} / \mathrm{ml}$ protein and reacted with click chemistry reagents (TCEP, TBTA, and $\mathrm{CuSO}_{4}$ ) and rhodamine-azide for $1 \mathrm{hr}$ at room temperature. Samples were separated by SDS-PAGE using a Tris-glycine system with an 11-cm separating gel. The gel was analyzed using a Hitachi FMBIOII fluorescence gel scanner, then stained with Coomassie blue for total protein and scanned at $700 \mathrm{~nm}$ using a LiCOR fluorescence gel scanner to demonstrate equivalent loading.

test whether any of these inhibitors have a role in stabilizing palmitoyl thioesters in different biological samples.

Early reported methods for bioorthogonal detection used azido fatty acids. Significant background labeling occurs in the presence of excess alkyne reporters, which is thought to be thiol dependent. It is therefore highly recommended to avoid azido fatty acids, since alkyne and strained-alkyne reporters demonstrate significantly higher nonspecific background labeling. Copper-catalyzed click chemistry is highly sensitive to amines, which are present in buffers such as Tris, so these buffers should be avoided. It is also essential to avoid buffers containing EDTA, which can affect the cop- per reactivity. When adding click chemistry reagents, it is recommended to add them individually to each tube, rather than making a master mix.

For more sensitive labeling, the concentration of rhodamine-azide can be increased at least ten-fold. For gel-based experiments, the typical final concentration of rhodamine-azide is $20 \mu \mathrm{M}$, whereas for proteomics experiments, the concentration of biotin-PEG3-azide required for saturated labeling was experimentally determined to be $500 \mu \mathrm{M}$ (Martin et al., 2012). During the click chemistry reaction, in the absence of any detergent, the proteome is precipitated by excess copper. Excess unreacted
Post-

Translational Modification: Specialized Applications

\subsection{7}


fluorophore can easily be removed by centrifugation and cold methanol washes. If the reaction is carried out in the presence of detergents, it can still be cleaned up by performing chloroform/methanol extractions (Wan et al., 2007).

Finally, when preparing samples for SDSPAGE, it is important to think about possible steps that could lead to thioester hydrolysis. $\beta$-Mercaptoethanol is known to exchange with thioesters, yet does not significantly affect 17-ODYA stability. On the other hand, DTT rapidly hydrolyzes palmitoyl thioesters. It is important to test gel-loading buffers with various reducing agents to ensure that palmitoyl thioesters are not sacrificed. It is also important to take precautions if boiling samples, since thioesters are highly susceptible to hydrolysis. Traditional Tris-glycine gels separate protein samples at $\mathrm{pH} 8.8$ or higher, which are not favorable conditions for thioester stability. Bis-Tris gels have several advantages over traditional Tris-glycine gels since they employ a neutral $\mathrm{pH}$. While these issues may be important under certain circumstances, the final separation and data quality are very similar for each type of gel chemistry when performing 17-ODYA in-gel fluorescence analysis.

\section{Anticipated Results}

Using the protocols described, protein palmitoylation can easily be analyzed by gelbased fluorescence. If a fluorescence flatbed gel scanner is not available, it is possible to transfer gels to membranes for streptavidin blots. Without pre-clearing, a significant number of endogenous biotinylated proteins will be detected in the absence of 17-ODYA. This is not the case with rhodamine-azide labeling. As discussed above, because fluorescent labeling is more sensitive, lower concentrations of fluorescent azide reporters are needed for detection (Fig. 14.15.2). This 200fold concentration difference likely accounts for the different proteins detected by biotinazide and rhodamine-azide. Overall, it is recommended that these concentrations be optimized individually for detection of specific palmitoylated proteins.

The turnover of protein palmitoylation can be detected using 17-ODYA pulse-chase labeling strategies (Fig. 14.15.3). Incorporation of 17-ODYA labeling reaches equilibrium after $2 \mathrm{hr}$. Replacing the 17-ODYA labeling medium at this point with excess palmitic acid for several hours reveals a subset of proteins with rapid palmitoylation turnover. Shorter label- ing periods show more significant turnover, but weaker signal. The total protein abundance does not change during the time course of the experiment, as shown by Coomassie staining.

\section{Time Considerations}

Metabolic labeling of cells with 17-ODYA takes several hours. Although the time is lengthened when performing a pulse-chase experiment, the entire protocol can still be easily accomplished in an afternoon. Preparing the cell pellet requires $\sim 30 \mathrm{~min}$. After lysing the cells by sonication, the membrane fraction is enriched by ultracentrifugation to enhance sensitivity by eliminating soluble proteins. This takes less than $1 \mathrm{hr}$. Further density fractionation is also compatible, so long as it is carried out in phosphate buffer with aminefree detergents; this could add significant time, depending on the fractionation protocol used. After protein quantification, the click chemistry reaction takes a few minutes to assemble and is left at room temperature for $1 \mathrm{hr}$. Following click chemistry, the sample is separated by SDS-PAGE and analyzed using a fluorescence gel scanner.

After harvesting the labeled cells, the cell pellets can be frozen at $-80^{\circ} \mathrm{C}$ for experiments on another day. Cells can be stored up to several months. It is preferred to complete the entire analysis after the initial thawing of the cell pellet to avoid freeze-thaw cycles.

\section{Literature Cited}

Ahearn, I.M., Tsai, F.D., Court, H., Zhou, M., Jennings, B.C., Ahmed, M., Fehrenbacher, N., Linder, M.E., and Philips, M.R. 2011. FKBP12 binds to acylated H-Ras and promotes depalmitoylation. Mol. Cell 41:173-185.

Charron, G., Zhang, M.M., Yount, J.S., Wilson, J., Raghavan, A.S., Shamir, E., and Hang, H.C. 2009. Robust fluorescent detection of protein fatty-acylation with chemical reporters. J. Am. Chem. Soc. 131:4967-4975.

Das, A.K., Bellizzi, J.J., III, Tandel, S., Biehl, E., Clardy, J., and Hofmann, S.L. 2000. Structural basis for the insensitivity of a serine enzyme (palmitoyl-protein thioesterase) to phenylmethylsulfonyl fluoride. J. Biol. Chem. 275:23847-23851.

Dekker, F.J., Rocks, O., Vartak, N., Menninger, S., Hedberg, C., Balamurugan, R., Wetzel, S., Renner, S., Gerauer, M., Schölermann, B., Rusch, M., Kramer, J.W., Rauh, D., Coates, G.W., Brunsveld, L., Bastiaens, P.I.H., and Waldmann, H. 2010. Small-molecule inhibition of APT1 affects Ras localization and signaling. Nat. Chem. Biol. 6:449-456. 
Drisdel, R.C. and Green, W.N. 2004. Labeling and quantifying sites of protein palmitoylation. Biotechniques 36:276-285.

Forrester, M.T., Hess, D.T., Thompson, J.W., Hultman, R., Moseley, M.A., Stamler, J.S., and Casey, P.J. 2011. Site-specific analysis of protein S-acylation by resin-assisted capture. J. Lipid Res. 52:393-398.

Hang, H.C., Geutjes, E.J., Grotenbreg, G., Pollington, A.M., Bijlmakers, M.J., and Ploegh, H.L. 2007. Chemical probes for the rapid detection of fatty-acylated proteins in mammalian cells. J. Am. Chem. Soc. 129:2744-2745.

Kang, R., Wan, J., Arstikaitis, P., Takahashi, H., Huang, K., Bailey, A.O., Thompson, J.X., Roth, A.F., Drisdel, R.C., Mastro, R., Green, W.N., Yates Iii, J.R., Davis, N.G., and El-Husseini, A. 2008. Neural palmitoyl-proteomics reveals dynamic synaptic palmitoylation. Nature 456:904909.

Kostiuk, M.A., Corvi, M.M., Keller, B.O., Plummer, G., Prescher, J.A., Hangauer, M.J., Bertozzi, C.R., Rajaiah, G., Falck, J.R., and Berthiaume, L.G. 2008. Identification of palmitoylated mitochondrial proteins using a bioorthogonal azido-palmitate analog. FASEB J. 22:721-732.

Martin, B.R. and Cravatt, B.F. 2009. Large-scale profiling of protein palmitoylation in mammalian cells. Nat. Methods 6:135-138.

Martin, B.R., Wang, C., Adibekian, A., Tully, S.E., and Cravatt, B.F. 2012. Global profiling of dynamic protein palmitoylation. Nat. Methods 9:84-89.
Roth, A.F., Wan, J., Bailey, A.O., Sun, B., Kuchar, J.A., Green, W.N., Phinney, B.S., Yates, J.R., III, and Davis, N.G. 2006. Global analysis of protein palmitoylation in yeast. Cell 125:1003-1013.

Rusch, M., Zimmermann, T.J., Burger, M., Dekker, F.J., Gormer, K., Triola, G., Brockmeyer, A., Janning, P., Bottcher, T., Sieber, S.A., Vetter, I.R., Hedberg, C., and Waldmann, H. 2011. Identification of acyl protein thioesterases 1 and 2 as the cellular targets of the Ras-signaling modulators palmostatin B and M. Angew Chem. Int. Ed. Engl. 50:9838-9842.

Schlesinger, M., Magee, A., and Schmidt, M. 1980. Fatty acid acylation of proteins in cultured cells. J. Biol. Chem. 255:10021-10024.

Schmidt, M.F., Bracha, M., and Schlesinger, M.J. 1979. Evidence for covalent attachment of fatty acids to Sindbis virus glycoproteins. Proc. Natl. Acad. Sci. U.S.A. 76:1687-1691.

Wan, J., Roth, A.F., Bailey, A.O., and Davis, N.G. 2007. Palmitoylated proteins: Purification and identification. Nat. Protoc. 2:15731584.

Yount, J.S., Moltedo, B., Yang, Y.-Y., Charron, G., Moran, T.M., López, C.B., and Hang, H.C. 2010. Palmitoylome profiling reveals S-palmitoylation-dependent antiviral activity of IFITM3. Nat. Chem. Biol. 6:610614.

Zhang, M.M., Tsou, L.K., Charron, G., Raghavan, A.S., and Hang, H.C. 2010. Tandem fluorescence imaging of dynamic S-acylation and protein turnover. Proc. Natl. Acad. Sci. U.S.A. 107:8627-8632.
Post-

Translational Modification: Specialized Applications

14.15.9 